Marquette University

e-Publications@Marquette

7-1-2012

Spectral Properties of H-Reflex Recordings After an Acute Bout of Whole-Body Vibration

Kristof Kipp

Marquette University, kristof.kipp@marquette.edu

Samuel T. Johnson

Oregon State University

Mark A. Hoffman

Oregon State University

Accepted version. Journal of Strength and Conditioning Research, Vol. 26, No. 7 (2012): 1915-1919.

DOI. (C) 2012 Lippincott Williams \& Wilkins, Inc. Used with permission.

This is not the final published version. 


\title{
Spectral Properties of H-Reflex Recordings after an Acute Boot of Whole-Body Vibration
}

\author{
Kristof Kipp \\ Department of Physical Therapy, Marquette University \\ Milwaukee, WI \\ Sam T. Johnson \\ Department of Nutrition and Exercise Sciences \\ Oregon State University \\ Corvallis, OR \\ Mark A. Hoffman \\ Department of Nutrition and Exercise Sciences \\ Oregon State University \\ Corvallis, OR
}

\begin{abstract}
Although research supports the use of whole-body vibration (WBV) to improve neuromuscular performance, the mechanisms for these improvements remain unclear. The purpose of this study was to identify the effect of WBV on the spectral properties of electrically evoked H-reflex recordings in the soleus (SOL) muscle. The $\mathrm{H}$-reflex recordings were measured in the SOL muscle of 20 participants before and after a bout of WBV. The H-reflexes were evoked every 15 seconds for 150 seconds after WBV. A wavelet procedure was used to extract spectral data, which were then quantified with a principle components analysis. Resultant principle component scores were used for statistical analysis. The analysis extracted 1 principle component associated with the intensity of the myoelectric spectra


NOT THE PUBLISHED VERSION; this is the author's final, peer-reviewed manuscript. The published version may be accessed by following the link in the citation at the bottom of the page.

and 1 principle component associated with the frequency. The scores of the principle component that were related to the myoelectric intensity were smaller at 30 and 60 milliseconds after WBV than before WBV. The WBV transiently decreased the intensity of myoelectric spectra during electrically evoked contractions, but it did not influence the frequency of the spectra. The decrease in intensity likely indicates a smaller electrically evoked muscle twitch response, whereas the lack of change in frequency would indicate a similar recruitment pattern of motor units before and after WBV.

Key Words: neuromuscular, frequency, motor unit, vibration

\section{Introduction}

The use of whole-body vibration (WBV) training is a popular exercise modality used to enhance the performance of the neuromuscular system $(6,7,13,14)$. Research supports the use of WBV to increase strength and power after acute exposure $(4,10,11,14)$. Nevertheless, despite there being a plethora of applied studies, the underlying mechanisms responsible for improvements after WBV exposure remain unclear. Because a lack of mechanistic information may impede the optimization and application of WBV as an exercise modality, elucidation of such mechanisms is paramount.

Because WBV has acute positive effects on neuromuscular function, a primary mechanism that has been suggested for improved performance is the potentiation of neuromuscular function through either altered motor neuron pool excitability or motor unit recruitment patterns $(2,8,12)$. Although several studies have investigated the changes in motor neuron pool excitability after WBV $(2,8,12)$, the findings in these studies have been equivocal. The other potential mechanism that has been proposed to account for WBV-induced changes is an altered motor unit recruitment pattern. It is known that direct tendon vibration promotes the ability to generate high firing rates in high-threshold motor units (3). In addition, tendon vibration is suggested to alter the threshold of motor unit recruitment and result in more rapid muscle activation (15). Although based on tendon vibration rather than on WBV, these findings implicate altered motor unit recruitment patterns, specifically in high-threshold motor units, as a basis for observed changes in muscle activation after vibration exposure $(3,15)$. Little, however, is known about the effects of WBV on the recruitment patterns of motor units.

Although motor unit recruitment patterns are difficult to study, recent studies show that wavelet analysis of the electromyographic 
signal provides a means to study the recruitment patterns of motor units across a variety of different tasks $(9,18,19)$. In particular, when combined with principle component analysis, wavelet analysis can be used to determine the time-varying intensity and frequency of myoelectric signals during volitional or reflexively evoked contractions $(9,18,19)$. Electrically evoked reflex contractions (i.e., H-reflex) appear to preferentially recruit faster motor units with higher recruitment thresholds than those with mechanically evoked reflex contractions (i.e., Tendon tap) as evidenced by a shift toward higher myoelectric frequency spectra (18). In addition, these methods are also sensitive enough to differentiate between different types of twitch contractions (i.e., tendon tap vs. supramaximal twitch) based on the intensity of the myoelectric spectra (9). Collectively, the combination of principle component and wavelet analyses therefore captures the time-varying spectral properties of electromyographic signals and delineates between changes in spectral intensity and frequency content, which in turn have been linked to motor unit recruitment patterns $(9,18,19)$. Considering that these methods provide a viable approach to study the changes in motor unit recruitment patterns, application of these methods would provide a novel insight into mechanistic changes in neuromuscular function after WBV.

The purpose of this study was therefore to determine if an acute bout of WBV affects the spectral properties of $\mathrm{H}$-reflex recordings of the soleus (SOL) muscle. Because the variables derived from the combined wavelet and principle component analyses provide information about motor unit recruitment patterns, it was hypothesized that any such changes after WBV would be present in the time-varying intensity and frequency content.

\section{Methods}

\section{Experimental Approach to the Problem}

To determine the effects of WBV on the spectral properties of the myoelectric signal of $\mathrm{H}$-reflexes, percutaneous peripheral nerve stimulations were used to elicit the H-reflex in the SOL muscle of the participants during quiet standing before and after a brief bout of WBV. Wavelet and principle component analyses were used to quantify the changes in the time-varying myoelectric intensity and frequency of the H-reflex signal to determine the effect of WBV on motor unit recruitment patterns. In addition, baseline measures were compared 
NOT THE PUBLISHED VERSION; this is the author's final, peer-reviewed manuscript. The published version may be accessed by following the link in the citation at the bottom of the page.

with several recordings after WBV to assess the time course of WBVinduced neuromuscular changes.

\section{Subjects}

Twenty (11 men, 9 women) healthy young adults (27.4 \pm 4.4 years) who were recreationally active (Tegner Activity Level Scale $>=5$ ) and had no known neurological deficits were recruited for this study. The participants were asked to refrain from intense exercise on the day of the testing. Each participant signed a written informed consent document that was approved by the University's Institutional Review Board before participating in the study.

\section{Procedures}

Data Collection Procedures. Before the bout of WBV, 10 electrical stimulations were delivered to elicit and establish baseline $\mathrm{H}$ reflex values. Stimulations were delivered while the participants stood quietly with their hands hanging at their side. The participants were instructed to limit any extraneous head and limb movement. Baseline stimulations were made with 15-second interstimuli intervals. For the WBV, the participants stood quietly, with the same posture as during the reflex recordings, on a vibration plate for 5 minutes (TurboSonic, Hood River, OR, USA). The WBV plate was set to vibrate at a frequency of $25 \mathrm{~Hz}$ and at an amplitude of 2-4 mm. H-reflex stimulations and recordings started again immediately after the cessation of the 5-minute bout of WBV and were continued every 15 seconds for 150 seconds to a total of 10 recordings.

Electromyography. The electromyogram (EMG) of the SOL muscle was recorded with lubricated $\mathrm{Ag} / \mathrm{AgCl}$ surface-recording electrodes (Biopac Systems Inc., Goleta, CA, USA) at a frequency of $2,000 \mathrm{~Hz}$. Electrodes were placed in series along the midline of the muscle belly with an interelectrode distance of approximately $2 \mathrm{~cm}$. Hreflex responses were elicited with a stimulating electrode $\left(2 \mathrm{~cm}^{2}\right)$ that was placed in the popliteal fossa, and a carbon-rubber dispersal electrode $\left(3 \mathrm{~cm}^{2}\right)$ was placed just superior to the patella on the distal thigh (Biopac Systems Inc.). H-reflexes were elicited with an s88 Grass stimulator (Grass Technologies, West Warwick, RI, USA) that delivered a square wave pulse (1 milliseconds) to the tibial nerve while the participants were standing quietly in a normal posture (i.e., hands hanging at the side, knees slightly flexed). A condensed recruitment 
NOT THE PUBLISHED VERSION; this is the author's final, peer-reviewed manuscript. The published version may be accessed by following the link in the citation at the bottom of the page.

curve was used to identify the maximum $\mathrm{H}$-reflex and $\mathrm{M}$-wave. The stimulation intensity was then adjusted to produce an $\mathrm{H}$-reflex equal to $10 \%$ of the maximum $\mathrm{M}$-wave and remained at this setting for the duration of the study.

Data Processing. A wavelet analysis with subsequent principle component analysis was used to extract information about the spectral properties of the $\mathrm{H}$-reflex recordings from each individual before and after WBV $(9,18,19)$. Preliminary analysis indicated that the presence of stimulation artifact and small M-waves influenced the results of the wavelet analysis. All the recordings were therefore trimmed to exclude the first 20 milliseconds of the recorded EMG where the stimulation artifact and $\mathrm{M}$-wave would be present. This step left a 50 -millisecond sample of EMG data that was used for the remainder of the analysis. Given an approximate latency of 30-35 milliseconds for the H-reflex, the 'trimming' of the trial did not affect $\mathrm{H}$-reflex recording (Figure 1 ). The EMG data were resolved into the time-frequency domain with a wavelet technique developed in Matlab (The MathWorks, Inc. Natick, MA, USA) at the University of Colorado (http://atoc.colorado.edu/research/wavelets). This wavelet algorithm uses a Morlet wavelet to calculate the time-varying power or intensity across a range of frequencies for the duration of the signal (Figure 1). The time-varying power is then summed into a global wavelet spectrum that gives the myoelectric power or intensity across all the frequencies for the given signal. The global wavelet spectra (i.e., intensity spectra) from all the individuals were then transposed and then pooled into data matrix $A$ that consisted of 220 rows (20 subjects $\times 11$ recordings per subject [i.e., baseline average +10 postvibration]) and 29 columns (arbitrary number of down-sampled frequencies). As part of the principle component analysis, the unit eigenvectors and eigenvalues were then extracted from covariance matrix $B$ of the original data matrix $A(18,19)$. Principle components were retained for analysis if the given eigenvalues for an eigenvector were greater than 1 (i.e., unity). The extracted eigenvectors were then multiplied with each individual's global wavelet spectra. The summation of the multiplication products then gave a set of principle component scores that determined how much of each eigenvector (i.e., principle component) was present within a given global wavelet spectrum. Because the principle components are extracted through an orthogonal matrix decomposition method, they provide unique 
information about the spectral properties (e.g., magnitude and frequency) contained within the signal $(18,19)$.

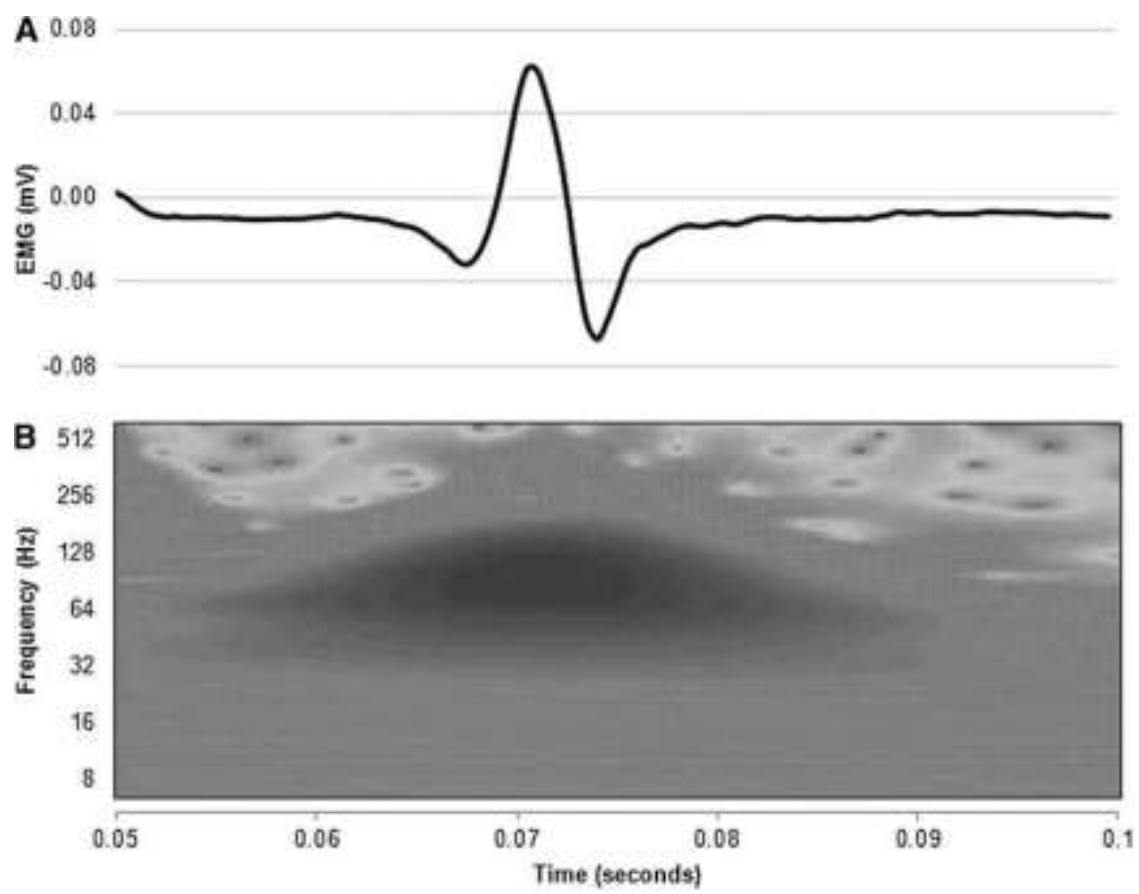

Figure 1. A representative trace of $(A)$ the electromyographic recording of an $\mathrm{H}$-reflex and $(B)$ the same signal after the time-frequency transform through the wavelet analysis. A darker color on the time-frequency graph indicates a greater intensity of the myoelectric signal. In addition, the location of the dark region provides information on the approximate time-varying frequency content of the myoelectric signal. Because the 2 extracted principle components (PCs) captured aspects related to the magnitude and frequency of the myoelectric signal, the changes in the PC scores could account for changes in the 'darkness' (i.e., intensity) or 'vertical shift' (i.e., frequency) of the tracing.

\section{Statistical Analyses}

Preliminary analyses with Kolmogorov-Smirnov tests were performed to check the probability distribution for all the variables. Violations of the normality in the data led to the use of nonparametric statistics. Friedman's test for comparison of multiple related samples was thus used to test for the overall differences in the time course among the extracted sets of each PC score. Post hoc follow-ups to a significant Friedman test were done with sign tests for 2 related samples to identify which post-WBV recording was different from baseline. The initial alpha level for significance testing with the Friedman tests was set to 0.05. A Bonferroni-corrected alpha level of 
0.005 was used to account for multiple comparisons $(n=10)$ between the pre-WBV baseline recordings and the 10 post-WBV recordings made at 15-second intervals (e.g., Pre-15sec, Pre-30sec ... Pre150sec). All analyses were run in SPSS Statistics 17.0 (IBM Corporation, Somers, NY, USA).

\section{Results}

The principle component analysis extracted 2 principle components that cumulatively explained $98.8 \%$ of the variance in the data. The first principle component explained $93.0 \%$ of the variance and had a positive weighting across all the frequencies, whereas the second principle component explained $5.2 \%$ of the variance and transitioned between a negative and positive weighting.

\begin{tabular}{|c|c|c|c|}
\hline \multirow[b]{2}{*}{ Time } & \multicolumn{2}{|c|}{ Principle component } & \multirow{13}{*}{$\begin{array}{l}\text { Table 1. Median ( } 25 \text { th to } 75 \text { th } \\
\text { percentile) scores for PC1 and PC2 } \\
\text { before (Pre) and after WBV (given at 15- } \\
\text { seconds intervals). *† }\end{array}$} \\
\hline & PC1 & $\mathrm{PC}_{2}$ & \\
\hline Pre & $0.16(0.07-0.28)$ & $0.03(0.01-052)$ & \\
\hline 15 & $0.07(0.03-0.28)$ & $0.01(0.01-0.03)$ & \\
\hline 30 & $0.05(0.03-0.12)$ & $0.01(0.01-0.01)$ & \\
\hline 45 & $0.04(0.03-0.13)$ & $0.01(0.01-0.02)$ & \\
\hline 60 & $0.06(0.02-0.16) \%$ & $0.01(0.01-0.03)$ & \\
\hline 75 & $0.09(0.02-0.14)$ & $0.01(0.01-0.03)$ & \\
\hline 90 & $0.06(0.04-0.19)$ & $0.01(0.01-0.28)$ & \\
\hline 105 & $0.07(0.02-0.22)$ & $0.01(0.01-0.03)$ & \\
\hline 120 & $0.06(0.01-0.15)$ & $0.01(0.01-0.03)$ & \\
\hline 135 & $0.12(0.03-0.22)$ & $0.01(0.01-0.06)$ & \\
\hline 150 & $0.08(0.03-0.16)$ & $0.01(0.01-0.03)$ & \\
\hline
\end{tabular}

The median scores (with 25th and 75th percentiles) for the first and second principle components across time are presented in Table 1. A significant chi-square statistic $\left(X^{2}=24.9 ; p=0.006\right)$ indicated an overall difference between the means for first principle component scores across time intervals. Post hoc analysis between individual means for first principle component scores indicated significant differences for the comparison Pre-30sec $(p=0.003)$ and Pre-60sec ( $p$ $=0.004)$. The chi-square statistic for the second principle component scores across time intervals indicated no overall difference between the means $\left(X^{2}=7.6 ; p=0.664\right)$.

\section{Discussion}

The purpose of this study was to determine if an acute bout of WBV affects the spectral properties of $\mathrm{H}$-reflex recordings of the SOL 
muscle. It was hypothesized that the changes in spectral properties, as measured through variables derived from wavelet and principle component analyses, would provide information about motor unit recruitment patterns after WBV. The analyses extracted 2 principle components that cumulatively explained $98 \%$ of the variance, which indicated that this technique was able to account for almost all of the spectral information contained within the myoelectric signals and therefore proved to be suitable to analyze spectral properties of the $\mathrm{H}$ reflex recordings. Moreover, the 2 extracted components were similar to those described for EMG signals during electrically and mechanically stimulated contractions (18) and during stationary cycling across a range of contraction intensities (19). The results indicated that WBV had a significant effect on the scores of the first principle component but not on the scores of the second principle component. Because the first and second principle components captured aspects related to the intensity and frequency of the myoelectric spectrum, respectively, these results indicate that the intensity, but not the frequency content of the myoelectric spectrum of H-reflex recordings, is affected by WBV. Because changes in myoelectric frequency spectra are related to the recruitment of motor units with different firing thresholds (18), it is unlikely that WBV affects the recruitment order of motor units.

The WBV transiently influenced the scores of the first principle component. In particular, the scores of the first principle component were significantly smaller 30 and 60 milliseconds after the cessation of WBV. Wakeling and Rozitis (18) found that a similar principle component was related to the intensity of muscle contraction. Specifically, these authors showed that a progressive increase in muscle activation during ramp contractions leads to a concomitant increase in the spectral intensity and the scores of the associated principle component (18). The first principle component therefore appeared to capture the intensity of the myoelectric signal, which indicated that the smaller scores after WBV point to a lower magnitude of the intensity of the signal's spectrum. Because an H-reflex recording essentially represents a muscle twitch response, it is likely that the vibration protocol used in this study led to a decrease in muscle twitch response and $\mathrm{H}$-reflex amplitude. Indeed several other studies have demonstrated that acute WBV is associated with a decrease in H-reflex amplitude $(2,8)$. Although contrary to reports of increased functional performance after WBV (e.g., vertical jump), it has recently been 
suggested that segmental spinal mechanisms (e.g., reduced presynaptic inhibition) may in fact overcompensate for the decrease in reflex excitability and thereby still potentiate muscular performance (8). The extent to which this is the case, however, still remains to be determined. The novel application of the techniques used in this study may facilitate future efforts that aim to study segmental spinal mechanisms after WBV.

The analyses also indicated that WBV did not influence the scores for the second principle component. This principle component captured a transition between a positive and negative weighting, Wakeling and Rozitis (18) found that a similar principle component can be used to describe the main frequency components of the myoelectric intensity spectra because greater scores of this component are associated with a higher frequency content of the spectra. Because higher myoelectric frequencies correspond to greater recruitment of faster motor units with higher recruitment thresholds $(9,18,19)$, a change in the scores of such a principle component would indicate an altered motor unit recruitment strategy. The lack of effect of WBV on the scores of the second principle component, however, indicated that the frequency content of the intensity spectra remained unchanged after WBV. It is consequently unlikely that WBV affects the recruitment order of motor units during electrically evoked contractions. In contrast, it should be noted that tendon vibration may increase the recruitment of high-threshold motor units $(3,15)$. The discrepancy in the effects of these modes of vibration should therefore caution researchers and practitioners from generalizing between the mechanism and outcomes associated with the respective type of vibration.

A general limitation to the conclusions made in this study relates to the chosen vibration settings for the WBV protocol. Because the vibration amplitude and frequency used in WBV protocols have differential effects on the functional response $(1,5)$, it may well be that exposure to WBV at other frequencies or amplitudes has differential effects on the frequency content of the myoelectric spectra.

Delineating between the differential effects of such 'programmingvariables' on neuromuscular measures would subsequently appear to be an important consideration in future studies. Furthermore, the $\mathrm{H}$ reflex recordings during this study were made during quiet standing. Because H-reflexes are subject to task-dependent modulation $(16,17)$, 
it would be of interest to investigate spectral properties under more dynamic situations than in standing or lying postures. Indeed, the application of these methods to dynamic tasks (e.g., jumping) similar to those in applied studies, which examine the effects of WBV on neuromuscular performance, would provide a much needed insight and improve the understanding of the neuromuscular effects of WBV. The aforementioned limitations therefore warrant additional research and provide future direction on the examination of mechanisms associated with changes in neuromuscular performance after WBV.

\section{Practical Applications}

The optimal application of WBV as an exercise modality depends on a thorough mechanistic understanding of the underlying neuromuscular responses and their effects on functional performance. The analyses used in this study helped characterize the intensity and frequency of myoelectric signals of the SOL muscle in response to an acute bout of WBV. Together, these variables identified the neuromuscular aspects related to motor unit recruitment strategies. The results indicated that only the intensity, but not the frequency content of the myoelectric spectrum, was affected by WBV. Specifically, the intensity of the myoelectric spectrum was depressed at various time points during the first minute after the WBV protocol. Therefore, it appears that the acute WBV protocol used in this study was associated with a short-term decrease in the intensity of electrically evoked muscle contraction. The recruitment of motor units during electrically evoked contractions, however, does not appear to be altered after WBV. Assessing the neuromuscular function after an 'optimal' WBV protocol and during dynamic paradigms should be at the center of future efforts by coaches and researchers who aim to study the efficacy of WBV as an exercise modality.

\section{Acknowledgements}

The authors wish to thank Jeffrey Doeringer for help with data collection.

\section{References}

1. Adams JB, Edwards D, Serravite DH, Bedient AM, Huntsman E, Jacobs KA, Del Rossi G, Roos BA, Signorile JF. Optimal frequency, displacement, duration, and recovery patterns to maximize power output following acute whole-body vibration. J Strength Cond Res 23: 237-245, 2009. 
NOT THE PUBLISHED VERSION; this is the author's final, peer-reviewed manuscript. The published version may be accessed by following the link in the citation at the bottom of the page.

2. Armstrong WJ, Nestle HN, Grinnell DC, Cole LD, Van Gilder EL, Warren GS, Capizzi EA. The acute effect of whole-body vibration on the Hoffmann reflex. J Strength Cond Res 22: 471-476, 2008.

3. Bongiovanni LG, Hagbarth KE. Tonic vibration reflexes elicited during fatigue from maximal voluntary contractions in man. J Physiol 423: 1$14,1990$.

4. Cormie P, Deane RS, Triplett NT, McBride JM. Acute effects of whole-body vibration on muscle activity, strength, and power. J Strength Cond Res 20: 257-261, 2006.

5. Di Giminiani R, Manno R, Scrimaglio R, Sementilli G, Tihanyi J. Effects of individualized whole-body vibration on muscle flexibility and mechanical power. J Sports Med Phys Fitness 50: 139-151, 2009.

6. Issurin VB. Vibrations and their applications in sport. A review. J Sports Med Phys Fitness 45: 324-336, 2005.

7. Jordan MJ, Norris SR, Smith DJ, Herzog W. Vibration training: An overview of the area, training consequences, and future considerations. J Strength Cond Res 19: 459-466, 2005.

8. Kipp K, Johnson ST, Doeringer JR, Hoffman MA. Spinal reflex excitability and homosynaptic depression after a bout of whole-body vibration. Muscle Nerve 43: 259-262, 2011.

9. Lee SS, de Boef Miara M, Arnold AS, Biewener AA, Wakeling JM. EMG analysis tuned for determining the timing and level of activation in different motor units. J Electromyogr Kinesiol, 21: 557-565, 2011.

10. Marin PJ, Rhea MR. Effects of vibration training on muscle power: A metaanalysis. J Strength Cond Res 24: 871-878, 2010.

11. Marin PJ, Rhea MR. Effects of vibration training on muscle strength: A meta-analysis. J Strength Cond Res 24: 548-556, 2010.

12. McBride JM, Nuzzo JL, Dayne AM, Israetel MA, Nieman DC, Triplett NT. Effect of an acute bout of whole body vibration exercise on muscle force output and motor neuron excitability. J Strength Cond Res 24: $184-189,2010$.

13. Nordlund MM, Thorstensson A. Strength training effects of whole-body vibration? Scand J Med Sci Sports 17: 12-17, 2007.

14. Rhea MR, Kenn JG. The effect of acute applications of whole-body vibration on the iTonic platform on subsequent lower-body power output during the back squat. J Strength Cond Res 23: 58-61, 2009.

Journal of Strength and Conditioning Research, Vol. 26, No. 7 (July 2012): pg. 1915-1919. DOI. This article is (c) Lippincott Williams \& Wilkins, Inc. and permission has been granted for this version to appear in e-Publications@Marquette. Lippincott Williams \& Wilkins, Inc. does not grant permission for this article to be further copied/distributed or hosted elsewhere without the express permission from Lippincott Williams \& Wilkins, Inc. 
NOT THE PUBLISHED VERSION; this is the author's final, peer-reviewed manuscript. The published version may be accessed by following the link in the citation at the bottom of the page.

15. Romaiguere $P$, Vedel JP, Pagni S. Effects of tonic vibration reflex on motor unit recruitment in human wrist extensor muscles. Brain Res 602: 3240, 1993.

16. Stein RB, Thompson AK. Muscle reflexes in motion: How, what, and why? Exerc Sport Sci Rev 34: 145-153, 2006.

17. Tokuno CD, Garland SJ, Carpenter MG, Thorstensson A, Cresswell AG. Sway-dependent modulation of the triceps surae $\mathrm{H}$-reflex during standing. J Appl Physiol 104: 1359-1365, 2008.

18. Wakeling JM, Rozitis AI. Spectral properties of myoelectric signals from different motor units in the leg extensor muscles. J Exp Biol 207: 2519-2528, 2004.

19. Wakeling JM, Uehli K, Rozitis AI. Muscle fibre recruitment can respond to the mechanics of the muscle contraction. J R Soc Interface 3: 533$544,2006$. 